\title{
Open source flood simulation with a 2D discontinuous-elevation hydrodynamic model
}

\author{
G. Davies $^{\mathrm{a}}$ and S. Roberts ${ }^{\mathrm{b}}$ \\ ${ }^{a}$ Community Safety Group, Geoscience Australia \\ ${ }^{\mathrm{b}}$ Mathematical Sciences Institute, Australian National University \\ Email: gareth.davies@ga.gov.au
}

\begin{abstract}
A new finite volume algorithm to solve the two dimensional shallow water equations on an unstructured triangular mesh has been implemented in the open source ANUGA software, which is jointly developed by the Australian National University and Geoscience Australia. The algorithm supports discontinuouselevation, or 'jumps' in the bed profile between neighbouring cells. This has a number of benefits compared with previously implemented continuous-elevation approaches. Firstly it can preserve lake-at-rest type stationary states with wet-dry fronts without using any mesh porosity type treatment (mesh porosity treatments allow the bed to absorb some water as though it were porous). It can also simulate very shallow frictionally dominated flow down sloping topography, as typically occurs in direct-rainfall flood models. In the latter situation, mesh porosity type treatments lead to artificial storage of mass in cells and associated mass conservation issues, whereas continuous-elevation approaches with good performance on shallow frictionally dominated flows tend to have difficulties preserving stationary states near wet-dry fronts. The discontinuous-elevation approach shows good performance in both situations, and mass is conserved to a very high degree, consistent with floating point error.
\end{abstract}

A further benefit of the discontinuous-elevation approach, when combined with an unstructured mesh, is that the model can sharply resolve rapid changes in the topography associated with e.g. narrow prismatic drainage channels, or buildings, without the computational expense of a very fine mesh. The boundaries between such features can be embedded in the mesh using break-lines, and the user can optionally specify that different elevation datasets are used to set the elevation within different parts of the mesh (e.g. often it is convenient to use a raster digital elevation model in terrestrial areas, and surveyed channel bed points in rivers).

The discontinuous-elevation approach also supports a simple and computationally efficient treatment of river walls. These are arbitrarily narrow walls between cells, higher than the topography on either side, where the flow is controlled by a weir equation and optionally transitions back to the shallow water solution for sufficiently submerged flows. This allows modelling of levees or lateral weirs which are much finer than the mesh size.

A number of benchmark tests are presented illustrating these features of the algorithm. All these features of the model can be run in serial or parallel, on clusters or shared memory machines, with good efficiency improvements on 10s-100s of cores depending on the number of mesh triangles and other case-specific details.

Keywords: Shallow water equations, finite volume methods, discontinuous-elevation, flood modelling 
G. Davies and S. Roberts, Open source flood simulation with a 2D discontinuous-elevation hydrodynamic ...

\section{INTRODUCTION}

Numerical solutions of the two-dimensional shallow water equations are widely used to simulate inundation caused by floods and tsunamis over realistic topography (e.g. Schubert et al., 2008; Jakeman et al., 2010; Costabile et al., 2013). The numerical quality of these inundation predictions is important, as they provide key hazard information to inform disaster risk estimates and risk reduction measures (e.g. de Bruijn et al., 2014; McLuckie et al., 2014). Accurate numerical solution of the shallow water equations is not straightforward, with numerical schemes often having difficulties with mass conservation, simulation of supercritical flow without ad-hoc modification of the flow equations, and maintaining numerical stability (especially at wet-dry fronts, over steep slopes, and for very shallow flows) (e.g. de Almeida et al., 2012; ARR, 2012; Smith and Wasko, 2012; Costabile et al., 2013; Sampson et al., 2013).

Finite volume schemes have become increasingly popular for the solution of the shallow-water equations because they are well adapted to simulate both subcritical and supercritical flows, and their flux-based formulation naturally promotes momentum and mass conservation (ARR, 2012; Kesserwania and Wang, 2014). ANUGA is an open source finite volume shallow water equations solver, and various versions of the software have been used for flood and tsunami simulation (Nielsen et al., 2005; Jakeman et al., 2010; Mungkasi et al., 2013; Mungkasi and Roberts, 2013). ANUGA can run in parallel on distributed or shared memory machines, and can efficiently use hundreds of cores on sufficiently large problems (Roberts et al., 2013). Here we describe a new finite volume scheme which has been implemented in ANUGA and is based on a discontinuous-elevation approach. Compared with previous solvers implemented in ANUGA it has the following advantages: 1) It preserves stationary states even at complex wet-dry fronts, and the same algorithm can model very shallow flow down coarsely resolved sloping topography; 2) It allows sharp changes in the topography to be represented using a relatively coarse mesh; 3) It admits a simple extension for modelling 'riverwalls' (thin weirs between two cells). Several examples illustrating these strengths of the new scheme are reported.

\section{EQUATIONS AND NUMERICAL METHOD}

\subsection{Finite-volume discretization of the shallow water equations}

The shallow water equations are:

$$
\frac{\partial \mathbf{U}}{\partial t}+\frac{\partial \mathbf{F}}{\partial x}+\frac{\partial \mathbf{G}}{\partial y}=\mathbf{R}^{\mathbf{z}}+\mathbf{R}^{\mathbf{f}}
$$

where:

$$
\begin{aligned}
\mathbf{U} & =[w, u h, v h] ; \quad \mathbf{F}=\left[u h, u^{2} h+g h^{2} / 2, u v h\right] ; \quad \mathbf{G}=\left[v h, u v h, v^{2} h+g h^{2} / 2\right] \\
\mathbf{R}^{\mathbf{z}} & =\left[0,-g h \frac{\partial z}{\partial x},-g h \frac{\partial z}{\partial y}\right] ; \quad \mathbf{R}^{\mathbf{f}}=\left[0,-g h S_{f x},-g h S_{f y}\right]
\end{aligned}
$$

and $x, y$ are spatial coordinates $(\mathrm{m}), t$ is time $(\mathrm{s}), w(x, y, t)$ is the stage (water surface elevation) with respect to an arbitrary vertical datum $(\mathrm{m}), h(x, y, t)$ is the flow depth $(\mathrm{m}),(u(x, y, t), v(x, y, t))$ are the $x$ and $y$ components of the flow velocity vector $(\mathrm{m} / \mathrm{s}), z(x, y)$ is the bed elevation $(\mathrm{m})$, and $g$ is a constant gravitational accelleration $\left(9.81 \mathrm{~m} / \mathrm{s}^{2}\right)$. Clearly $w=h+z$. The friction slope terms $S_{f x}$ and $S_{f y}$ drive momentum losses due to friction, and are modelled with standard Manning friction closures:

$$
S_{f x}=\eta^{2} \frac{u \sqrt{u^{2}+v^{2}}}{h^{4 / 3}} ; \quad S_{f y}=\eta^{2} \frac{v \sqrt{u^{2}+v^{2}}}{h^{4 / 3}}
$$

where $\eta(x, y)$ is a Manning's roughness coefficient which is related to the sub-mesh scale roughness of the land surface and the flow.

To numerically solve Equation 1 we discretize the model domain with an unstructured triangular mesh. At any instant in time the real flow state inside the $i$ 'th mesh triangle $\triangle_{i}$ may vary spatially, but the average flow state can be represented by a three-dimensional vector:

$$
\mathbf{U}_{\mathbf{i}}=\left[w_{i},(u h)_{i},(v h)_{i}\right]:=\frac{1}{A_{i}} \iint_{\triangle_{i}} \mathbf{U} d x d y
$$


G. Davies and S. Roberts, Open source flood simulation with a 2D discontinuous-elevation hydrodynamic ...

where $A_{i}$ is the area of $\triangle_{i}$.

Integrating Equation 1 over $\triangle_{i}$ and applying the divergence theorem to the spatial derivative terms leads to a system of ordinary differential equations for the $\mathbf{U}_{\mathbf{i}}$ :

$$
\frac{d \mathbf{U}_{\mathbf{i}}}{d t}+\frac{1}{A_{i}} \sum_{j=1}^{3} l_{i j}\left(\mathbf{F}_{\mathbf{i j}} n_{i j}^{x}+\mathbf{G}_{\mathbf{i j}} n_{i j}^{y}\right)=\mathbf{R}_{\mathbf{i}}^{\mathbf{z}}+\mathbf{R}_{\mathbf{i}}^{\mathbf{f}}
$$

where the use of an $i j$ subscript denotes a quantity on the $j$ 'th edge of $\triangle_{i}, j \in(1,2,3), l_{i j}$ is the edge length, $\mathbf{F}_{\mathbf{i j}}, \mathbf{G}_{\mathbf{i j}}$ are the spatially averaged values of $\mathbf{F}$ and $\mathbf{G}$ on the edge, $\mathbf{n}_{\mathbf{i j}}=\left(n_{i j}^{x}, n_{i j}^{y}\right)$ is the outward unit normal vector to the edge, and $\mathbf{R}_{\mathbf{i}}^{\mathbf{z}}, \mathbf{R}_{\mathbf{i}}^{\mathbf{f}}$ are the spatially averaged values of $\mathbf{R}^{\mathbf{z}}$ and $\mathbf{R}^{\mathbf{f}}$ respectively on $\triangle_{i}$. Although there are no numerical approximations in Equation 5 its solution requires evaluation of the flux terms $\mathbf{F}_{\mathbf{i j}}$ and $\mathbf{G}_{\mathbf{i j}}$, and the source terms $\mathbf{R}_{\mathbf{i}}^{\mathbf{z}}$ and $\mathbf{R}_{\mathbf{i}}^{\mathbf{f}}$. In practice these must be approximated using the known $\mathbf{U}_{\mathbf{i}}$.

\subsection{Approximation of the flux and source terms}

To compute the flux and source terms we require values of flow variables along the edge of every triangle. These are computed using a discontinuous piecewise linear interpolation, e.g. for the edge stage $w_{i j}$ :

$$
w_{i j}=w_{i}+r \nabla \mathbf{w}_{\mathbf{i}} \cdot\left(\mathbf{x}_{\mathbf{i j}}-\mathbf{x}_{\mathbf{i}}\right)
$$

where $\mathbf{x}_{\mathbf{i j}}=\left(x_{i j}, y_{i j}\right)$ is the edge midpoint coordinate, $\mathbf{x}_{\mathbf{i}}=\left(x_{i}, y_{i}\right)$ is the centroid coordinate, $\nabla \mathbf{w}_{\mathbf{i}}$ is equal to the gradient of the plane containing the centroid stages of the three triangles neighbouring $\triangle_{i}$, and $r \in[0,1]$ is a slope limiter computed to prevent any edge stage from overshooting the centroid stages in the triangle and its neighbours (e.g. Kesserwania and Wang, 2014).

Interpolations similar to Equation 6 are applied to estimate the edge stage $w_{i j}$, depth $h_{i j}$, and velocities $u_{i j}, v_{i j}$. The edge bed elevation is then $z_{i j}=w_{i j}-h_{i j}$. At each edge every variable attains two distinct values because the interpolation is discontinuous, and hence the elevation is also discontinuous at the triangle edges (Audusse et al., 2004).

To compute the flux on a given edge, consider a rotated coordinate system where the $x$ axis is directed along the outward normal to the edge as viewed from $\triangle_{i}$. With this coordinate system, let $w_{i j}, h_{i j}, u_{i j}^{\prime}, v_{i j}^{\prime}, z_{i j}$ be the edge variables extrapolated from one neighbouring triangle, and $w_{n m}, h_{n m}, u_{n m}^{\prime}, v_{n m}^{\prime}, z_{n m}$ the corresponding variables extrapolated from the other triangle sharing the edge. The prime superscript is used when the new coordinate system has changed the value of the variable. Following Audusse et al. (2004), define:

$$
z^{*}=\max \left(z_{i j}, z_{n m}\right) ; \quad h_{i j}^{*}=\max \left(h_{i j}-z^{*}+z_{i j}, 0\right) ; \quad h_{n m}^{*}=\max \left(h_{n m}-z^{*}+z_{n m}, 0\right)
$$

The flux through the edge as evaluated naïvely from quantities on the $i j$ edge in the rotated coordinate system is:

$$
\mathcal{H}_{\mathrm{ij}}^{\prime}=\left[u_{i j}^{\prime} h_{i j}^{*},\left(u_{i j}^{\prime}\right)^{2} h_{i j}^{*}+\frac{g}{2}\left(h_{i j}^{*}\right)^{2}, u_{i j}^{\prime} v_{i j}^{\prime} h_{i j}^{*}\right]
$$

and we similarly define $\mathcal{H}_{\mathrm{nm}}^{\prime}$ using the quantities on the $n m$ edge. These are combined to produce a single edge flux $\mathbf{H}_{\mathbf{i} \mathbf{j}}^{\prime}$ with a HLL Riemann solver:

$$
\mathbf{H}_{\mathbf{i j}}^{\prime}=\frac{s_{+} \mathcal{H}_{\mathbf{i j}}^{\prime}-s_{-} \mathcal{H}_{\mathbf{n m}}^{\prime}+s_{+} s_{-}\left(\mathbf{U}_{\mathbf{n m}}^{\prime}-\mathbf{U}_{\mathbf{i j}}^{\prime}\right)}{s_{+}-s_{-}}
$$

where:

$$
\begin{aligned}
s_{+} & =\max \left(u_{i j}^{\prime}+\sqrt{g h_{i j}^{*}}, u_{n m}^{\prime}+\sqrt{g h_{n m}^{*}}, 0 .\right) ; s_{-}=\min \left(u_{i j}^{\prime}-\sqrt{g h_{i j}^{*}}, u_{n m}^{\prime}-\sqrt{g h_{n m}^{*}}, 0 .\right) \\
\mathbf{U}_{\mathbf{i j}}^{\prime} & =\left[w_{i j}, u_{i j}^{\prime} h_{i j}^{*}, v_{i j}^{\prime} h_{i j}^{*}\right] ; \quad \mathbf{U}_{\mathbf{n m}}^{\prime}=\left[w_{n m}, u_{n m}^{\prime} h_{n m}^{*}, v_{n m}^{\prime} h_{n m}^{*}\right]
\end{aligned}
$$

The flux $\mathbf{H}_{\mathbf{i j}}^{\prime}$ is rotated back to the $x, y$ coordinate system and used in place of the term $\left(\mathbf{F}_{\mathbf{i j}} n_{i j}^{x}+\mathbf{G}_{\mathbf{i j}} n_{i j}^{y}\right)$ in Equation 5. Note the rotated $\mathbf{H}_{\mathbf{i j}}^{\prime}$ flux will be identical to the rotated $\mathbf{H}_{\mathbf{n m}}^{\prime}$ flux, so the flux computations conserve mass and momentum. 
G. Davies and S. Roberts, Open source flood simulation with a 2D discontinuous-elevation hydrodynamic ...

The friction related source term $\mathbf{R}_{\mathbf{i}}^{\mathbf{f}}$ in Equation 5 is approximated using pointwise values of $\mathbf{U}_{\mathbf{i}}$ at centroids. The bed slope source term $\mathbf{R}_{\mathbf{i}}^{\mathbf{z}}$ in Equation 5 is approximated to be well balanced as (Audusse et al., 2004):

$$
\mathbf{R}_{\mathbf{i}}^{\mathbf{z}}=\frac{g}{2 A_{i}} \sum_{j=1}^{3} l_{i j}\left(\left(h_{i j}^{*}\right)^{2}-h_{i j}^{2}-\left(h_{i j}+h_{i}\right)\left(z_{i j}-z_{i}\right)\right)
$$

\subsection{Time integration and velocity clipping}

Equation 5 is integrated in time using an explicit 2nd order Runge-Kutta scheme. Variations of the algorithm have also been successfully implemented using first order explicit euler time-stepping, but they are not considered further herein. The timestep is chosen based on the CFL condition as described in Roberts et al. (2013).

The flow depth in every $\triangle_{i}$ is checked in every timestep. If it is less than $1 \times 10^{-5} \mathrm{~m}$ then the cell's depth integrated velocities $(u h)_{i},(v h)_{i}$ are set to zero. Without this we find computations may produce very high velocities in extremely shallow flows, as has been widely reported elsewhere (e.g. Nikolos and Delis, 2009; Costabile et al., 2013).

\section{Properties of The discontinuous-elevation SCHEME}

\subsection{Well balancing}

The scheme described above is well balanced (i.e. it can preserve stationary states), even over complex topography with wet-dry fronts. Figure 1 gives an example of complex topography with a stationary wet-dry front, where Manning's $\eta$ ranges from 0.02 to 0.04 depending on land-use (Smith and Wasko, 2012). To demonstrate the scheme's well-balanced nature we computed the flow in this scenario for $100 \mathrm{~s}$ ( $\sim 3000$ timesteps $)$. Analytically the flow should be stationary. Consistent with this the computed instantaneous flow speed remains effectively zero throughout the simulation $\left(<2 \times 10^{-12} \mathrm{~m} / \mathrm{s}\right)$. Early ANUGA algorithms could not preserve this stationary state over complex topography (Davies, 2011). The latter paper proposed a porosity-type flow algorithm which can solve this problem, but cannot successfully model shallow flow down coarsely resolved sloping topography (Section 3.2).

To test the discontinuous-elevation scheme's robustness when water is flowing off the buildings (as would happen in a direct-rainfall simulation with embedded buildings), at $t=10 \mathrm{~s}$ we added $1 \mathrm{~cm}$ of water to buildings within the initially wet areas, and computed the evolution of the flow for $3000 \mathrm{~s}$. Considering that flow off building tops can generate instabilities in some numerical schemes (ARR, 2012; Sampson et al., 2013), we check: 1) whether instabilities occur as water flows off the buildings into the initially stationary pond, and; 2) whether the model returns to a stationary state. The computed instantaneous maximum flow speed peaks at $0.4 \mathrm{~m} / \mathrm{s}$ early in the simulation and then steadily decays with no obvious instabilities (Figure 1). It reaches $<2 \times 10^{-4} \mathrm{~m} / \mathrm{s}$ after $3000 \mathrm{~s}$, consistent with a return to stationary state. The water volume change was $4 \times 10^{-11} \mathrm{~m}^{3}$, indicating good mass conservation as compared with the ideal value of zero.

\subsection{Coarsely resolved sheet flow}

Direct rainfall flood models will almost inevitably include some upper-catchment areas exhibiting friction dominated sheet flow. Often the model is coarsely resolved so that the elevation change in a single cell is large compared to the flow depth. This can cause problems for some numerical methods. For example, if the edge depth is computed by subtracting the real edge bed elevation from an edge stage computed with first order extrapolation, then it will be far too large and result in unrealistic fluxes. Also, porosity-type wetting and drying approaches (Davies, 2011) will incorrectly absorb a potentially large part of the discharge. Similarly some numerical methods employ values of the threshold depth for setting velocities to zero that are much larger than the value used here $\left(1 \times 10^{-5} \mathrm{~m}\right)$, which means that significant amounts of water may be artificially stored (ARR, 2012).

To examine the current scheme's performance for coarsely resolved sheet flow, we simulate flow down a uniformly sloping plane ( $x$ length $400 \mathrm{~m}, y$ length $100 \mathrm{~m}$ ) with gradient of $-1 / 10$ in the $x$ direction and a Manning coefficient of 0.03 . The mesh triangles have an area of $100 \mathrm{~m}^{2}$ (side lengths ranging from 14-20 m) so the elevation within a single triangle drops $\simeq 1.4$ to $2 \mathrm{~m}$. We impose a small discharge of $0.1 \mathrm{~m}^{3} / \mathrm{s}$ uniformly along the upstream boundary $(x=0)$. The analytical solution may be computed assuming the flow becomes steady and uniform $\left(S_{f x}=-\frac{\partial z}{\partial x}\right)$ and leads to flow depths slightly below $4 \mathrm{~mm}$ which is much 
G. Davies and S. Roberts, Open source flood simulation with a 2D discontinuous-elevation hydrodynamic ...
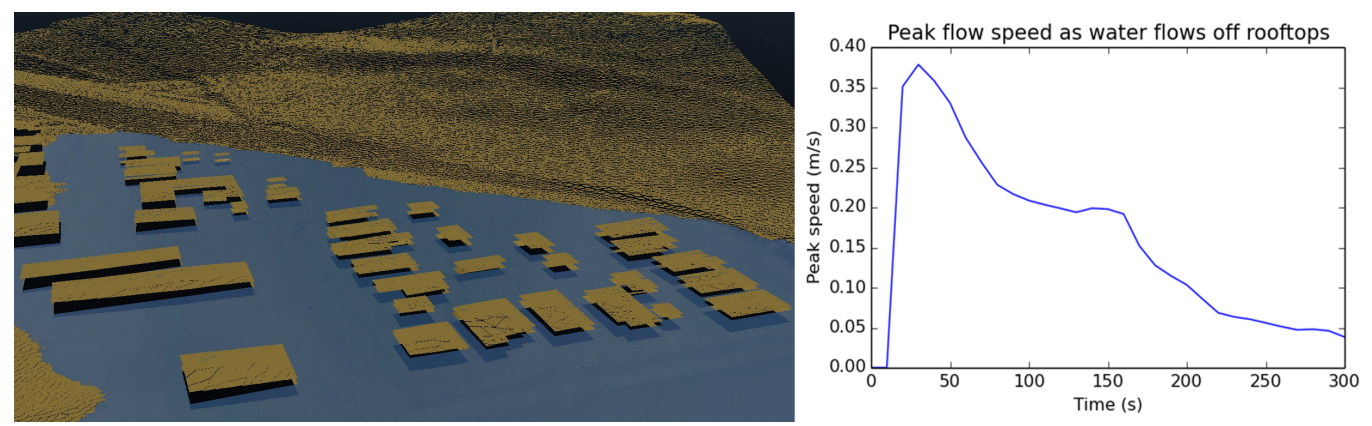

Figure 1. Left: Topography and initial condition for the well balancing test case. Blue areas are wet with a constant stage, and beige areas are dry. Note the discontinuities in the elevation at buildings, which emerge from the water. The buildings were produced by adding $3 \mathrm{~m}$ to the topography inside polygons defining the building-footprint. Right: Temporal evolution of instantaneous peak flow speed in the entire model domain after a perturbation to the balanced state. $1 \mathrm{~cm}$ of water was added to all buildings in the wet region of the figure, which then flows off the buildings into the ponded areas.
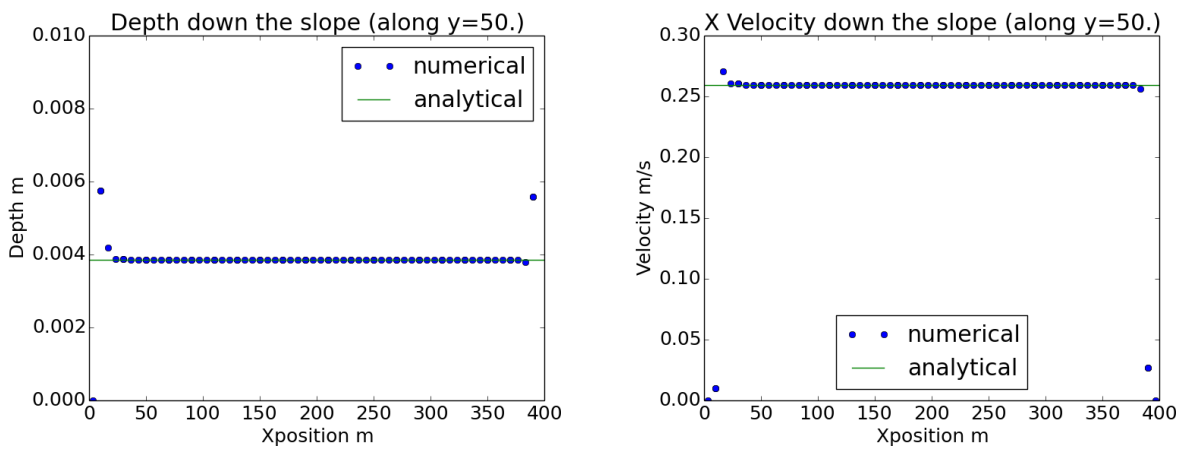

Figure 2. Numerical and analytical flow depth (left) and velocity (right) in the downstream direction for the coarsely resolved sheet flow example. Centroid values for all triangles within $10 \mathrm{~m}$ of the line $y=50$ are plotted. Flow is from left to right. Deviations from the analytical solution near the boundaries are explained in the text.

less than the elevation range of the mesh triangles. Figure 2 shows the depth and velocity computed by the model after $3000 \mathrm{~s}$ agrees well with the analytical solution away from the boundaries. Deviations occur at the upstream boundary because the flow has not had time to reach a steady-uniform state, and at the downstream boundary because a reflective boundary condition was imposed leading to water ponding (for visual clarity the plot range excludes the high depths there), while the model is highly accurate in the steady-uniform region.

\subsection{Sharp resolution of changes in topography}

A key benefit of discontinuous-elevation is that it permits the sharp resolution of rapid changes in topography, even with a coarse mesh. For example, Figure 3 shows a channel with rectangular cross-section defined sharply using only 1-2 cells along the channel width. The mesh is constructed using 'breaklines' to define the channel banks at a cross-channel distance of $10 \mathrm{~m}$ and $20 \mathrm{~m}$ (Figure 3). Breaklines are lines that mesh triangles cannot cross, so each breakline is covered by triangle edges. Although this example has a particularly idealised geometry (to facilitate analytical solution), irregular channel shapes can also be modelled straightforwardly using irregular 2D breaklines. With continuous-elevation solvers it is impossible to represent such channels with steep banks at coarse resolution. A fine mesh can be used to resolve the topography arbitrarily well in a continuous-elevation solver, but this is not always feasible as the computational effort increases rapidly with mesh refinement.

We compare the analytical solution for overbank flow with numerical solutions using a coarse mesh (10 m 
G. Davies and S. Roberts, Open source flood simulation with a 2D discontinuous-elevation hydrodynamic ...
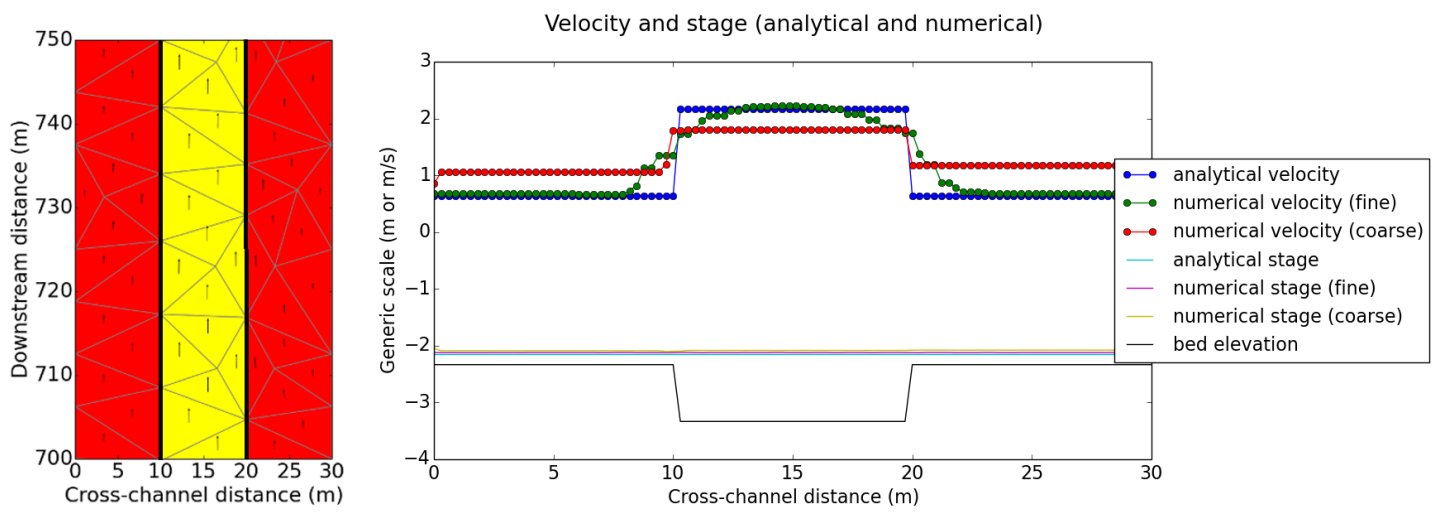

Figure 3. Left: Coarse-mesh discontinuous-elevation representation of a prismatic channel (yellow triangles) flanked by narrow floodplains (red triangles). Breaklines (bold black lines) separate the channel and the floodplains. Velocity vectors are depicted as black arrows. The full channel is $1000 \mathrm{~m}$ long (not shown). Right: The analytical stage and velocities over a cross-section with overbank flow are compared to those computed with a coarse-mesh $(\sim 1$ triangle over the channel width) and a fine-mesh $(\sim 6-8$ triangles over the channel width). The channel slope $=1 / 300, \eta=0.03$, and the discharge is $28.16 \mathrm{~m}^{3} / \mathrm{s}$.

triangles with $\simeq 1$ triangle over the channel width $)$ and a fine mesh $(1.5 \mathrm{~m}$ triangles with $\simeq 6-8$ triangles over the channel width) (Figure 3 ). Theoretically the latter requires around $(10 / 1.5)^{3} \simeq 300$ times more computational effort than the former. Both numerical solutions exhibit numerical diffusion of momentum around the banks, causing them to deviate from the analytical solution (Figure 3). Numerical diffusion tends to decrease velocities in the channel, increase velocities on the floodplains, and produce higher overall channel drag (Figure 3). However, both solutions give reasonably accurate depth predictions (errors of $6 \mathrm{~cm}$ with the coarse mesh and $2 \mathrm{~cm}$ with the fine mesh). While it is typically preferable to use a fine mesh if the computational effort is not excessive, for the discontinuous-elevation algorithm the coarse-mesh errors may be practically acceptable in some field situations, considering that the unknown Manning's roughness will have a greater effect on the channel's conveyance and will require calibration.

With a small modification, the discontinuous-elevation algorithm can be extended to allow 'thin' walls of arbitrary height to be inserted on the edges between two mesh triangles. This allows riverwalls or levees which are much finer than the mesh size to be represented in the model. To do this the variable $z^{*}$ (Equation 7) is set equal to the wall height prior to the flux computation, which prevents any flow over the wall until it is overtopped. Numerically, both mesh triangles behave as though their neighbour has the higher bed elevation. If the wall is overtopped, the computed flux is re-scaled so that it agrees with the flux computed from a widely used weir equation (Villemonte, 1947), with a transition back to the shallow water solution when the weir is sufficiently submerged. We will report on tests of this approach in a future publication.

\section{CONCLUSIONS}

The ANUGA code (https://github.com/GeoscienceAustralia/anuga_core) includes about 30 validation tests covering analytical, experimental and realistic examples, which can be used to more thoroughly evaluate the performance of the above flow algorithm (termed 'DE1' in ANUGA). While it is not possible to discuss all these tests herein, in our experience with these and other applications the above scheme is quite robust and accurate compared with the continuous-elevation approaches implemented in ANUGA. Particular advantages of the discontinuous-elevation approach include that: the scheme is well balanced even at complex wet-dry fronts, while also being able to simulate frictionally dominated shallow flows over coarsely resolved sloping topography; it can sharply resolve rapid changes in the topography with a relatively coarse mesh; and it admits a straightforward extension for modelling riverwalls. The main limitation we are aware of is in simulating frictionless wet-dry fronts, for which the algorithm tends to predict that drying fronts dry more slowly than in analytical solutions. This behaviour has been reported for another finite-volume solver using a similar discontinuous-elevation flux discretization (Kesserwania and Wang, 2014). We do not expect this to be highly relevant for field applications, since very shallow flows over realistic topography will tend to be frictionally dominated. 
G. Davies and S. Roberts, Open source flood simulation with a 2D discontinuous-elevation hydrodynamic ...

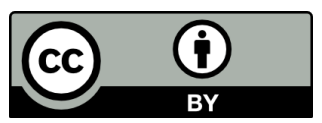

This paper is published with the permission of the CEO of Geoscience Australia.

\section{REFERENCES}

ARR (2012). Revision project 15: Two dimensional modelling in urban and rural floodplains (draft report). In Australian Rainfall and Runoff. Engineers Australia.

Audusse, E., F. Bouchut, M.-O. Bristeau, R. Klein, and B. Perthame (2004). A fast and stable well-balanced scheme with hydrostatic reconstruction for shallow water flows. SIAM Journal of Scientific Computing 25(6), 2050-2065.

Costabile, P., C. Costanzo, and F. Macchione (2013). A storm event watershed model for surface runoff based on $2 \mathrm{~d}$ fully dynamic wave equations. Hydrological Processes 27, 554-569.

Davies, G. (2011). A well-balanced discretization for a shallow water inundation model. In 19th International Congress on Modelling and Simulation, Perth, Australia, 1216 December 2011.

de Almeida, G. A. M., P. B. J. E. Freer, and M. Souvignet (2012). Improving the stability of a simple formulation of the shallow water equations for 2-d flood modeling. Water Resources Research 48(W05528).

de Bruijn, K. M., F. Diermanse, and J. Beckers (2014). An advanced method for flood risk analysis in river deltas, applied to societal flood fatality risk in the netherlands. Natural Hazards and Earth System Science 14, 2767-2781.

Jakeman, J., O. Nielsen, K. Van Putten, R. Mleczko, D. Burbidge, and N. Horspool (2010). Towards spatially distributed quantitative assessment of tsunami inundation models. Ocean Dynamics.

Kesserwania, G. and Y. Wang (2014). Discontinuous galerkin flood model formulation: luxury or necessity? Water Resources Research 50, 6522-6541.

McLuckie, D., M. Babister, G. Smith, and R. Thomson (2014). Updating national guidance on best practice flood risk management. In Floodplain Management Association National Conference.

Mungkasi, S. and S. G. Roberts (2013). Validation of anuga hydraulic model using exact solutions to shallow water wave problems. In Journal of Physics: Conference Series, Volume 423.

Mungkasi, S., R. van Drie, and S. G. Roberts (2013). Predictions on arrival times of water of the st. francis dam break flood using anuga. In 20th International Congress on Modelling and Simulation, Adelaide, Australia, 16 December 2013.

Nielsen, O., S. Roberts, D. Gray, A. McPherson, and A. Hitchman (2005). Hydrodynamic modelling of coastal inundation. In A. Zerger and R. Argent (Eds.), MODSIM 2005 International congress on modelling and simulation, pp. 518-523.

Nikolos, I. and A. Delis (2009). An unstructured node-centered finite volume scheme for shallow water flows with wet/dry fronts over complex topography. Comput. Methods Appl. Mech. Engrg. 198, 3723-3750.

Roberts, S., Y. Oishi, and M. Li (2013). High resolution tsunami inundation simulations. In 20th International Congress on Modelling and Simulation, Adelaide, Australia, 16 December 2013.

Sampson, C. C., P. D. Bates, J. C. Neal, and M. S. Horritt (2013). An automated routing methodology to enable direct rainfall in high resolution shallow water models. Hydrological Processes 27, 467-476.

Schubert, J. E., B. F. Sanders, M. J. Smith, and N. G. Wright (2008). Unstructured mesh generation and landcover-based resistance for hydrodynamic modeling of urban flooding. Advances in Water Resources 31, $1603-1621$.

Smith, G. and C. Wasko (2012). Revision project 15: Two dimensional simulations in urban areas - representation of buildings in 2d numerical flood models. In Australian Rainfall and Runoff. Engineers Australia.

Villemonte, J. (1947). Submerged weir discharge studies. Engineering News Record 866, 54-57. 